\title{
Evaluación de costes en hemodiálisis
}

\section{Eduardo Garoé Fernández García}

Fundación Hospital Calahorra. Calahorra. La Rioja

\section{Resumen}

Existe un desconocimiento del coste económico de la hemodiálisis y de los distintos tratamientos depurativos renales, por lo que el objetivo de este trabajo fue valorar económicamente el tratamiento depurador en función de la técnica empleada y el acceso vascular utilizado.

El valor medio de la sesión es de $62,79 €$, que incluye desde la hemodiálisis convencional de alto flujo $(45,19 €)$ a la Acetate Free Biofiltration $(79,35 €)$. Con respecto al acceso vascular, el coste medio por sesión fue de $4,31 €$; en los pacientes portadores de catéter fue de $5,35 €$ y de $3,28 €$, en los portadores de fístula arteriovenosa interna o injerto.

Por otro lado, el impacto económico de nuestra unidad en la Fundación Hospital Calahorra, supone el $5,08 \%$ del total, correspondiendo el $41 \%$ al gasto en personal, el $33 \%$ al de material fungible y el $15 \%$ al gasto farmacéutico.

\section{PALABRAS CLAVE:}

- HEMODIÁLISIS

- COSTES

- TÉCNICAS DIALITICAS

- IMPACTO ECONÓMICO

Correspondencia:

Eduardo Garoé Fernández García

C/Tirso de Molina, $n^{\circ} 14-6^{\circ} \mathrm{B}$

26003 Logroño.

egfernandez@fhcalahorra.com.

\section{Evaluation of costs in haemodialysis}

\section{Abstract}

There is a lack of knowledge of the economic cost of haemodialysis and of the different kidney depurative treatments, and therefore the aim of this work was to evaluate the depurative treatment economically on the basis of the technique applied and vascular access used.

The average cost of the session is $62.79 €$, which includes from conventional high-flow haemodialysis $(45.19 €)$ to Acetate Free Biofiltration $(79.35 €)$. As regards vascular access, the average cost per session was $4.31 €$; in patients with a catheter it was $5.35 €$ and $3.28 €$ in patients with an open arteriovenous fistula or graft.

In addition, the economic impact of our unit in the Fundación Hospital Calahorra represents 5.08\% of the total, of which $41 \%$ relates to staff costs, $33 \%$ to fungible material and $15 \%$ to pharmaceutical expense.

\section{KEY WORDS}

- HAEMODIALYSIS

- COSTS

- DIALYTIC TECHNIQUES

- ECONOMIC IMPACT 


\section{Introducción}

La evolución científica, el desarrollo de nuevas técnicas de diálisis, así como las diferentes mejoras, encaminadas a mejorar la calidad en el tratamiento depurativo renal de los pacientes con IRC, ha supuesto un notable cambio en el mundo de la Nefrología en los últimos años ${ }^{1}$.

Ante el escaso conocimiento del coste económico de la hemodiálisis en los centros públicos ${ }^{2}$ y dada la variedad de tratamientos existentes, nos surge la inquietud de valorar económicamente el coste de cada sesión, en función de los diferentes tipos de técnicas que aplicamos a nuestros pacientes, aún con la complejidad dada de la multitud de variables existentes en hemodiálisis ${ }^{3}$.

\section{Objetivo}

1. Acercarnos al coste más exacto posible, en función de las distintas técnicas dialíticas y de las peculiaridades más relevantes de los pacientes como el tipo de acceso vascular.

2. Objetivar el consumo global de nuestra unidad.

\section{Material y Método}

Hemos realizado un estudio descriptivo, a través de la valoración de todos los gastos imputados a nuestra unidad a lo largo del año 2006.

La obtención de los datos, se ha realizado por medio del volcado de los mismos, facilitados por los procesos soporte (almacén, farmacia y hostelería) a una hoja de cálculo Excel, teniendo en cuenta el cuaderno de mando de la Fundación Hospital Calahorra.

- La Unidad de Hemodiálisis dispone de la siguiente dotación de monitores: Monitores Bellco Formula ®: 14 ( 3 de reserva)

- Monitores Hospal INTEGRA ®: 2

- Monitor Gambro AK 200 Ultra S ®: 1.

- Planta de Ósmosis Inversa GAMBRO CWP 100 ®.

- Planta de Ósmosis GAMBRO WRO 62 ®.
La dotación de personal es de:

- 4 Auxiliares de Enfermería.

- 5 DUE.

- 3 Facultativos Especialistas en Nefrología.

- 1 Coordinador de Enfermería.

Los pacientes se distribuyen en 4 turnos distintos, repetidos 3 veces por semana, de lunes a sábado. Nuestra unidad atendió a lo largo del 2006 a 37 pacientes en tratamiento de IRC, con un total de 5196 sesiones de HD, de las cuales la distribución media de pacientes por tipo de técnica ha sido la siguiente (tabla 1):

- HD Convencional Bellco ®: $56,7 \%$.

- HD Convencional Hospal ß: 5,4\%.

- Biofiltración: 10,8\%.

- HDF 0n-Line: 10,8\%.

- AFB: $16,21 \%$.

\begin{tabular}{lccccc} 
Técnica & $\begin{array}{c}\text { HD } \\
\text { Convencional } \\
\text { BELLCO }\end{array}$ & $\begin{array}{c}\text { HD } \\
\text { Convencional } \\
\text { HOSPAL }\end{array}$ & Biofiltración & HDF Online & AFB \\
\hline $\begin{array}{l}N^{0} \\
\text { Pacientes }\end{array}$ & 21 & 2 & 4 & 4 & 6 \\
\hline$\%$ & $56^{\prime} 7 \%$ & $5^{\prime} 4 \%$ & $10^{\prime} 8 \%$ & $10^{\prime} 8 \%$ & $16^{\prime} 21 \%$ \\
\hline
\end{tabular}

Tabla 1. Distribución de pacientes por técnicas

La distribución media de pacientes según el acceso vascular (tabla 2) es:

- Catéter: $24.3 \%$.

- FAVI o Goretex: 75.7\%.

\begin{tabular}{|c|c|}
\hline Tipo Acceso Vascular & No Pacientes \\
\hline Catéter & 9 \\
\hline FAVI o Goretex & 28 \\
\hline
\end{tabular}

Tabla 2. Distribución de pacientes por tipo de acceso vascular

El estudio se encamino en dos direcciones:

1. Conocer el consumo generado, en función del tipo de técnica dialítica empleada.

2. Conocer el impacto económico de la Unidad de Diálisis dentro de la Fundación Hospital Calahorra. 
Para conocer el consumo generado en función del tipo de técnica dialítica, se elaboró un listado en hoja de cálculo Excel por cada tipo de terapia, con todo el material fungible utilizado en la técnica, detallando cantidades y se le aplicó el importe concreto. Se completó, adjuntando datos referentes a cada paciente concreto, aplicando el importe y haciendo la media del total entre todos los pacientes tratados con la técnica. Además, se calculo el gasto, en función del acceso vascular utilizado (catéter o FAVI).

Por otro lado para conocer el impacto económico de la Unidad de Diálisis dentro de la Fundación Hospital Calahorra, y completar el estudio, se solicitó a los procesos soporte, los importes económicos globales de productos farmacéuticos y mantenimiento de instalaciones (preventivo y correctivo) y se obtuvo el coste del personal de las tablas salariales, por medio del convenio vigente en nuestro hospital.

Todos esos datos fueron posteriormente prorrateados en función de las 5196 sesiones realizadas en la unidad en el año 2006, con lo que se obtuvo el coste económico medio por sesión de HD.

\section{Resultados}

La información obtenida de este estudio, desveló la siguiente distribución del coste por tipo de técnica y sesión (figura 1):

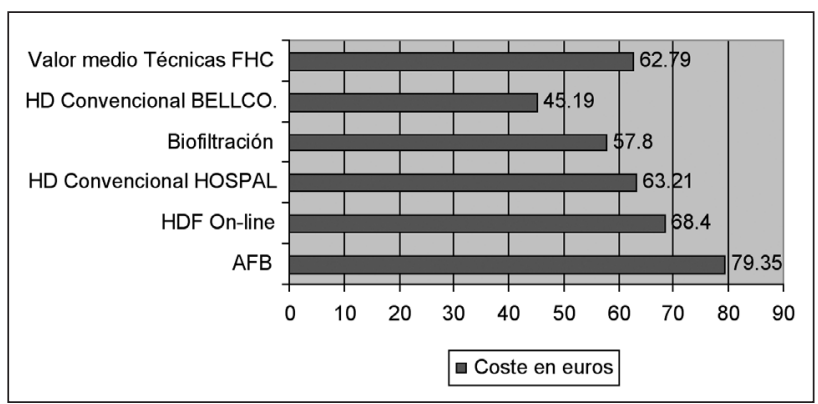

Figura 1. Distribución del coste por tipo de técnica y sesión

- AFB: $79,35 €$.

- HDF 0n-line: $68,40 €$.

- HD Convencional HOSPAL: 63, $21 €$.

- Biofiltración: 57,80€.

- HD Convencional BELLC0: 45,19 €.

- El valor medio de las técnicas empleadas en la FHC es de $62,79 €$.
El tipo de acceso vascular del paciente, suponen un coste añadido:

- Portador de catéter: 5,35 €.

- Portadores de FAVI o Goretex: 3,28 €.

- Consumo medio independiente del acceso vascular: 4,31 €. Esta cantidad, supondría el coste medio del material empleado en conexión y desconexión, independientemente de cual sea el tipo de acceso vascular del paciente.

El coste medio de cada sesión de HD, asociada al material fungible, independientemente del tipo de técnica y del tipo de acceso vascular asciende de este modo a $67,10 €$.

Al estudiar el impacto económico de la unidad de $\mathrm{HD}$, dentro la FHC, se obtuvieron los siguientes datos (figura 2):

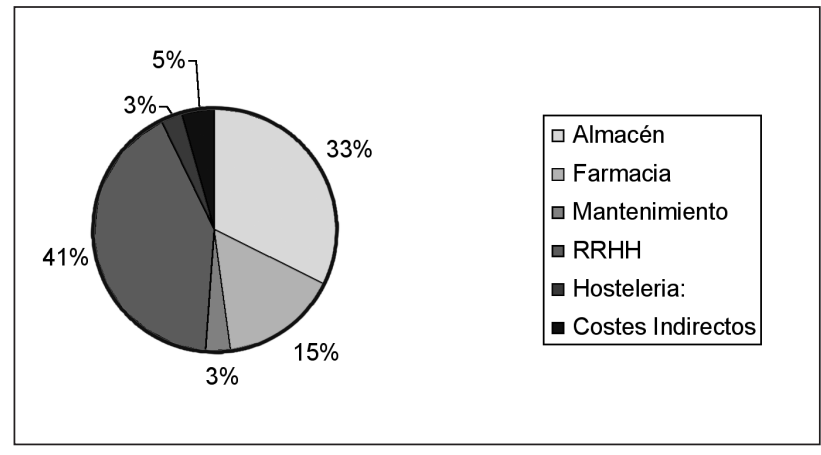

Figura 2. Distribución de costes de la unidad de HD

- Almacén: El material fungible utilizado por la unidad de diálisis en el año 2006, ascendió a $308.125 €$.

- Farmacia: El gasto farmacéutico, supuso $144.929 €$.

- Mantenimiento: Tanto el mantenimiento correctivo, como el preventivo alcanzó los $31.165 €$.

- RRHH: La suma neta de salarios del personal de la unidad fue de $393.854 €$.

- Hosteleria: Cocina y limpieza ascendieron a $9.141 €$ y $15.283 €$, respectivamente.

- Costes Indirectos: Los costes indirectos, como la telefonía, laboratorio, lavandería, seguridad, etc, ascendió a $43.307 €$.

- TOTAL: $945.804 €$, con un peso global de nuestra Unidad en la FHC del 5,08\%. 


\section{Discusión}

Una vez conocidos los importes de cada técnica, es evidente que algunas despuntan con costes más elevados, como la AFB o la Hemodiafiltración On-Line. Además, en cuanto al consumo global generado por nuestra unidad, los gastos más significativos son:

1. Recursos humanos: $41 \%$

2. Almacén: $33 \%$

3. Farmacia: $15 \%$

Es indudable que las técnicas especiales y la mejora de la calidad de las aguas suponen un impacto económico, pero también es cierto que esto redunda en beneficio de los pacientes. Nuestros indicadores de calidad indican un claro descenso de la tasa de hospitalización y fallecimientos en los años 2005 y 2006 con tendencia a estabilizarse o seguir disminuyendo (figura 3), esto coincide con la adquisición de una nueva planta de aguas que permite utilizar nuevas técnicas.

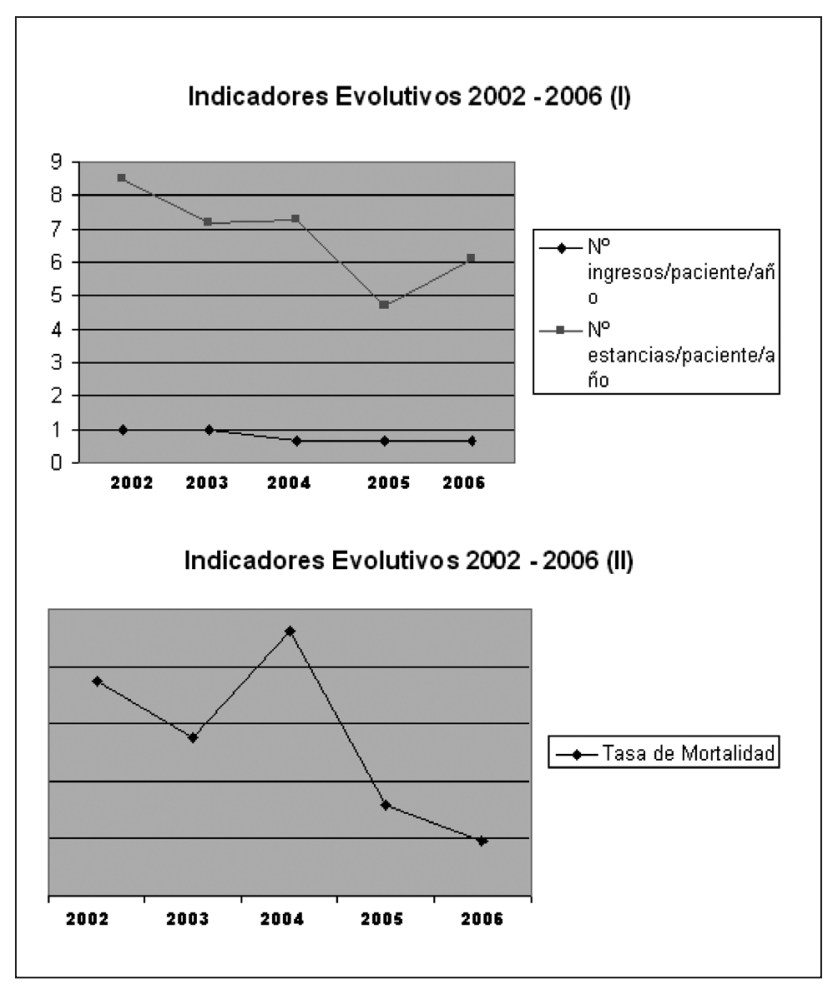

Figura 3. Indicadores evolutivos de ingresos y mortalidad $2002-2006$
Dado que existe una necesidad de utilizar eficientemente unos recursos escasos que satisfagan la demanda creciente, nos planteamos si la disponibilidad de estos tratamientos resultaría conveniente para todos los pacientes, teniendo en cuenta el incremento de gasto que conlleva. Esta disyuntiva nos lleva a plantear la cuestión: ¿Estaríamos preparados para trabajar dentro de un Comité de Bioética en el que se valorara la disponibilidad de esas técnicas para los pacientes, bajo criterios de justicia y equidad, optimizando así los recursos económicos, técnicos y humanos de nuestro colapsado sistema sanitario?".

Por último y como reflexión final, cada vez que conectamos a un paciente a nuestros monitores de $\mathrm{He}$ modiálisis, somos más conscientes y responsables del gasto que ello implica y esto es una pequeña contribución a nuestra calidad profesional.

\section{Bibliografía}

1. Remigio MJ. Pasado y Presente en Hemodiálisis. Página web de ALCER-Orense. Octubre 2006. Disponible en: http://www.mundourense.com/alcer-ourense/IVxornadas/pasadoypresente.ppt

2. Parra E, Porres JC, Blanco I. Inaceptable Desconocimientos de los Costes de la Hemodiálisis en Centros Públicos. Página web de la Asociación de Economía de la Salud. Junio 2007. Disponible en: http://www.aes.es/Jornadas/pdfs/25.pdf

3. Burgos R, Martín J, López del Amo MªJ, Arellano $J$, Pérez C, Pozo F. Importancia del método de estimación de costes en diálisis y trasplante renal. Revista de la Sociedad Española de Nefrología. 2001; XXI (Supl 4).

4. Pérez $R$, Rodríguez P. La calidad del líquido de Hemodiálisis. Página web del II Congreso Internacional de Nefrología por Internet. Noviembre 2001. Disponible en:

http://www.uninet.edu/cin2001/html/conf/perez/p erez.html.

5. Tanus E. La Bioética 20 años después. Revista Renal Argentina. 2001. 